\title{
Um Mapeamento Sistemático sobre o Ensino de Programação para Pessoas com Deficiência
}

\author{
Elaine Cristina Juvino de Araújo ${ }^{12}$, Wilkerson L. Andrade ${ }^{2}$ \\ ${ }^{1}$ Instituto Federal de Educação, Ciência e Tecnologia da Paraíba (IFPB) \\ Campina Grande - Paraíba - Brazil \\ ${ }^{2}$ Universidade Federal de Campina Grande (UFCG) \\ Campina Grande - Paraíba - Brazil \\ elainec@ifpb.edu.br,wilkerson@computacao.ufcg.edu.br
}

\begin{abstract}
There are many challenges faced by people with disabilities, among them are the challenges in learning programming. As there is a need to address deficiencies in a specific way in teaching and learning, a systematic mapping was carried out in order to provide an overview of the area. Initially, 1844 works were found, but only 29 met the inclusion and exclusion criteria of articles defined in this work. These works were classified according to their approach. As a result, a research map in the area was created and it was also identified that the greatest efforts have been made for people with visual impairments and that there is no standardized methodology for each type of disability.
\end{abstract}

Resumo. Muitos são os desafios enfrentados pelas pessoas com deficiência, entre esses destacam-se os desafios na aprendizagem de programação. Como existe a necessidade de se tratar as deficiências de maneira específica no ensinoaprendizagem, foi realizado um mapeamento sistemático a fim de fornecer uma visão geral da área. Inicialmente foram encontrados 1844 trabalhos, porém apenas 29 satisfizeram os critérios de inclusão e exclusão de artigos definidos. Estes trabalhos foram classificados de acordo com sua abordagem. Como resultado, um mapa de pesquisas na área foi criado e também foi identificado que os maiores esforços têm sido para pessoas com deficiência visual e que não existe uma padronização de metodologia para cada tipo de deficiência.

\section{Introdução}

Pode-se definir como deficiência qualquer condição incapacitante que acomete as pessoas, seja do tipo permanente como uma síndrome ou temporária como um braço imobilizado por uma fratura. Martin [Martin 2009] destaca que existem vários tipos de deficiência como: déficit intelectual, físico, visual, auditivo ou múltiplo (quando o indivíduo possui mais de um tipo de deficiência), as síndromes (como a de Down) e os transtornos (como o Autismo). No Brasil, segundo o último censo, 23,9\% da população declarou possuir algum tipo de deficiência. Essa significativa parcela da população apresenta inúmeros desafios, principalmente no que diz respeito à sua capacitação [IBGE 2010].

Atualmente, um dos maiores desafios na educação, não apenas do Brasil, é fazer com que estudantes com deficiência tenham acesso a ensino adequado (hoje eles são incluídos na escola e excluídos do conhecimento). O material didático geralmente 
não foi projetado para esta inclusão, sendo um motivo relevante para o desenvolvimento de ferramentas (digitais) e metodologias que considerem as diferentes necessidades de cada deficiência, mas que seja aplicável dentro de um contexto comum de sala de aula [Martin 2009].

Pessoas com deficiência podem entrar em qualquer curso técnico ou superior que desejar, inclusive cursos da área de Computação. Ao iniciar um curso dessa área, elas irão cursar disciplinas de programação, que compreendem o processo de concepção, criação e teste de algoritmos. Essa área já é considerada complexa e com altos índices de evasão, mesmo para alunos que não possuem deficiência alguma [Souza et al. 2016]. A abordagem utilizada nos cursos de programação envolve, muitas vezes, a exposição de situaçõesproblema nas quais o aluno deverá ser capaz de criar um algoritmo que chegue à solução esperada. Quando o aluno não consegue entender o problema que precisa resolver, dificilmente chegará a acertar o exercício. Gomes e Mendes [Gomes and Mendes 2015] expõem que os métodos de ensino mais tradicionais não são os mais adequados para apoiar a aprendizagem de programação, nas quais se apresentam turmas com número elevado de estudantes e pouca produtividade das aulas expositivas no campo da programação. Neste contexto, pode-se supor que a criação de conteúdos motivadores para a aprendizagem e a utilização de métodos de ensino adequados podem ser importantes para um suporte adequado aos estudantes, em particular aqueles com deficiência.

A área de ensino de programação para pessoas com deficiência ainda necessita de mais iniciativas que auxiliem docentes e discentes no processo de ensino-aprendizagem. Este trabalho mostra os resultados de um mapeamento sistemático focado em descobrir no que e como os pesquisadores estão trabalhando na área de ensino de programação voltada para pessoas com deficiência, o protocolo de pesquisa criado e a forma como foi realizado. O restante do trabalho está organizado da seguinte forma: a Seção 2 apresenta trabalhos relacionados, a Seção 3 descreve a metodologia para realização do mapeamento sistemático, a Seção 4 apresenta os resultados do estudo, na Seção 5 discute-se o que foi observado na pesquisa, incluindo prováveis ameaças à validade. Por fim, as considerações finais estão na Seção 6.

\section{Trabalhos Relacionados}

Al-Ratta e Al-Khalifa [Al-Ratta and Al-Khalifa 2013] executaram uma revisão sistemática de literatura para analisar e classificar trabalhos relacionados ao ensino de programação para pessoas cegas. Os resultados apontaram que a maioria das pesquisas focaram em soluções para permitir que pessoas cegas usassem tecnologias, porém poucas foram as pesquisas que tratavam sobre o ensino de programação para esse público. O mapeamento aqui apresentado se difere desse por ser mais abrangente, visto que o propósito é de identificar o que está sendo feito na área de ensino de programação sem focar em apenas uma deficiência, buscando catalogar as estratégias de ensino-aprendizagem atualmente utilizadas, além de selecionar trabalhos mais recentes entre 2010 e 2020.

Silva et al. [Silva et al. 2015] realizaram uma revisão sistemática de literatura sobre ensino-aprendizagem de programação em eventos nacionais, verificando que há uma prevalência de trabalhos voltados apenas para o ensino superior e não abordaram a temática para estudantes com deficiência. $\mathrm{O}$ trabalho aqui exposto realizou buscas de artigos apenas em Inglês, nacionais ou não, e não se limitou a um nível de ensino específico. 
IX Congresso Brasileiro de Informática na Educação (CBIE 2020)

Anais do XXXI Simpósio Brasileiro de Informática na Educação (SBIE 2020)

\section{Procedimentos Metodológicos}

A metodologia escolhida para identificar trabalhos relacionados ao tema foi o mapeamento sistemático de literatura (MSL). Esse método é recomendado para áreas de pesquisa em que existem poucos estudos primários relevantes e de qualidade, identificando lacunas para possíveis trabalhos futuros e problemas em aberto para serem investigados.

Para a execução do mapeamento, foi utilizada a abordagem definida por Petersen et al. [Petersen et al. 2008], que consiste em um conjunto de etapas bem definidas bastante difundido em pesquisas na área de Computação. A seguir, são descritas as etapas do processo escolhido e como foram realizadas.

\subsection{Definição das Questões de Pesquisa (QP) do Mapeamento Sistemático}

A primeira etapa do MSL consiste em identificar as prováveis questões de pesquisa a serem respondidas. A ideia nesse momento inicial é de definir questões que remetem ao problema de pesquisa de uma forma mais geral. Desejou-se com esse MSL identificar trabalhos nos seguintes aspectos: (i) abordagens associadas aos tipos específicos de deficiências; (ii) tecnologias que estão sendo utilizadas na área; (iii) limitações identificadas nas pesquisas recentes; (iv) problemas em aberto na área de adaptação de exercícios de programação para pessoas com deficiência; e (v) como estão sendo feitas as avaliações das pessoas com deficiência em disciplinas de programação.

Para esse trabalho foram identificadas as seguintes QP:

- QP1 - Quais as principais abordagens pedagógicas investigadas no domínio da Acessibilidade em Ensino de Programação?

- QP2 - Em quais níveis educacionais tem sido mais investigada a área de Acessibilidade em Ensino de Programação?

- QP3 - Quais as tecnologias utilizadas na Acessibilidade em Ensino de Programação?

- QP4 - Como os alunos deficientes são avaliados no Ensino de Programação?

- QP5 - Quais os problemas em aberto na Acessibilidade em Ensino de Programação?

\subsection{Escopo da Pesquisa}

Seguindo os passos definidos por Petersen [Petersen et al. 2008], após a identificação das QP, define-se o escopo da pesquisa. Nesta fase, são definidas a chave de busca e as bases nas quais os trabalhos serão pesquisados.

A primeira definição nesta etapa foi de que a busca seria feita apenas em Inglês e artigos publicados nos últimos 10 anos, de forma a identificar trabalhos mais relevantes e recentes na área. O passo seguinte foi a escolha das bases nas quais seriam localizados os trabalhos. Foram selecionadas as seguintes bases de busca: IEEE, ACM e Springer Link.

A definição da chave de busca é muito importante, pois é através dela que os trabalhos relevantes na área são localizados e catalogados. Foi definido que a pesquisa nas bases se daria apenas no título, resumo e palavras chave. Para esse projeto foi definida a seguinte chave de busca inicial: (("programming" OR "computer science" OR "cs") AND ("teaching" OR "exercise" OR "students") AND ( "disabilities" OR "autism" OR "blind" OR "deaf" OR “accessibility” OR "for all”)). Em uma primeira busca, foram encontrados 444 trabalhos na ACM, 678 na Springer e 722 no IEEE. A etapa seguinte foi a triagem dos artigos que efetivamente seriam relevantes para a pesquisa. 

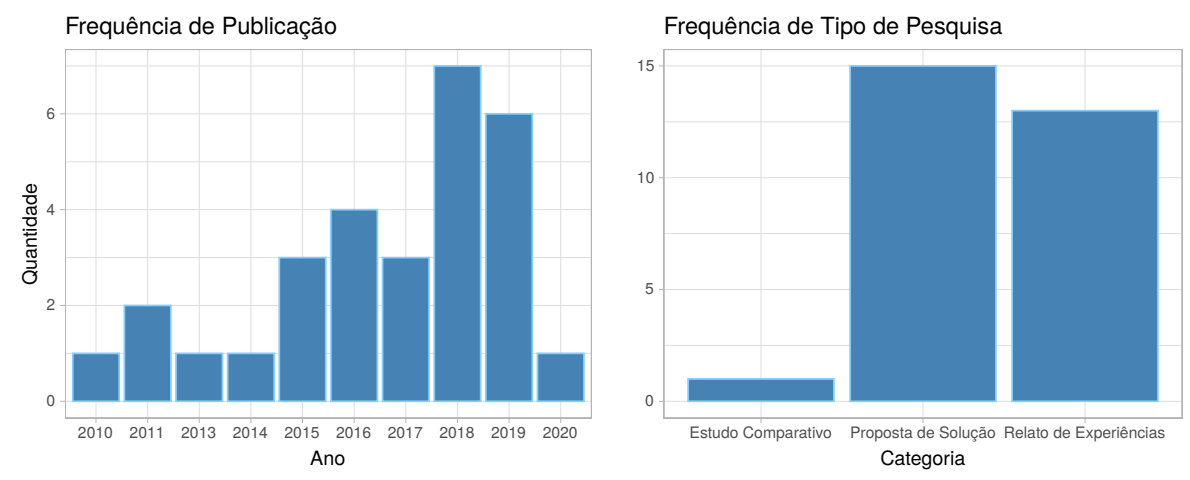

Figura 1. Frequência de (a) publicação e (b) de tipo de pesquisa

\subsection{Triagem dos Artigos}

De forma a buscar os trabalhos que são importantes para as questões de pesquisa, foram definidos alguns critérios de inclusão e exclusão. Como critérios de inclusão temos: CI1) Artigos em Inglês; CI2) Os trabalhos devem conter os termos da chave de busca presentes no título e/ou no resumo e/ou nas palavras-chave do artigo selecionado; CI3) Artigos completos que apresentem método, proposta ou prática de ensino de programação para pessoas com algum tipo de deficiência. Os critérios de exclusão definidos foram: CE1) Artigos que não descrevem um método, proposta ou prática de ensino de programação para pessoas com algum tipo de deficiência; CE2) Artigos com abordagens ou modelos provenientes de uma mesma pesquisa; CE3) Artigos de revisão de literatura.

Feitas as consultas às bases de dados, os trabalhos retornados foram refiltrados após a leitura do título, palavras chave e resumo de cada trabalhos, de forma a observar quais trabalhos atendiam aos critérios de inclusão e exclusão. Dos 1844 trabalhos, 425 foram considerados candidatos por atenderem ao CI1, após uma nova triagem foi feita a leitura dos resumos e reaplicação dos critérios de inclusão e exclusão, sendo selecionados 29 trabalhos ao final, cujos detalhes estão disponíveis online ${ }^{1}$.

\section{Resultados}

Esta pesquisa foi realizada no mês de Junho de 2020. Assim, esta seção apresenta os resultados que foram obtidos até essa data. Mostramos os dados de forma geral e em seguida, respondemos cada questão de pesquisa.

\subsection{Resultados Gerais}

O resultado do mapeamento sistemático realizado apontou que existe interesse em ensino de programação para pessoas com deficiência, conforme mostra a Figura 1(a). O ano com maior número de publicações foi 2018, com a publicação de 7 artigos. Com relação ao tipo de pesquisa realizada, os trabalhos foram catalogados segundo a classificação de Petersen [Petersen et al. 2008], que estabelece os seguintes tipos: relato de experiência, pesquisa de avaliação, pesquisa de validação, proposta de solução, artigo de opinião e artigo filosófico. Como pode-se ver na Figura 1(b), foram encontrados trabalhos em apenas 3 tipos, sendo proposta de solução o tipo mais frequente, seguido por relato de experiências.

\footnotetext{
${ }^{1}$ https://tinyurl.com/y6sr8sjk
} 
IX Congresso Brasileiro de Informática na Educação (CBIE 2020)

Anais do XXXI Simpósio Brasileiro de Informática na Educação (SBIE 2020)

\subsection{QP1 - Quais as principais abordagens pedagógicas investigadas no domínio da Acessibilidade em Ensino de Programação?}

As abordagens identificadas nos artigos catalogados neste mapeamento sistemático foram: Programação Adaptada (16/29 artigos), Programação em Blocos (4/29 artigos), Robótica (5/29 artigos), Programação Tátil ou Desplugada (2/29 artigos), Programação Colaborativa (1/29 artigo) e Jogos (1/29 artigo). Como pode-se ver, a abordagem mais utilizada foi o que chamamos de programação adaptada. Nessa abordagem é utilizada algum tipo de adaptação para o ensino de programação tradicional, por exemplo o uso de um leitor de telas para pessoas cegas ou a tradução de conteúdo para língua de sinais para pessoas surdas.

\subsection{QP2 - Em quais níveis educacionais tem sido mais investigada a área de Acessibilidade em Ensino de Programação?}

$\mathrm{Na}$ investigação realizada neste trabalho, foi identificado que existem pesquisas no ensino fundamental, médio e superior. Os níveis mais investigados são fundamental (14/29 artigos) e médio (11/29 artigos). Esse resultado pode ser explicado pela maioria dos trabalhos serem originados dos Estados Unidos onde existem iniciativas consistentes de inserção de aprendizagem de programação para alunos antes de entrarem no ensino superior.

Quando consideramos o contexto do nosso país vemos que não é comum ter contato com conceitos de programação antes de entrar em um curso de nível superior, o que coloca diversos alunos, inclusive com deficiência, em um curso específico da área mas com quase ou nenhuma base. Dessa forma, investigar as experiências de alunos com deficiência no ensino superior é uma área que pode ser abordada, já que foi percebida a pouca quantidade de trabalhos relacionados a esse nível de ensino.

\subsection{QP3 - Quais as tecnologias utilizadas na Acessibilidade em Ensino de Programação?}

O apoio de tecnologias é bastante utilizado no ensino de programação, porém foi observado no MSL que não existe um padrão quando se trata de pessoas com deficiência. A maioria das tecnologias abordadas foi utilizada por no máximo 2 estudos, o que pode ser visto na Tabela 1. Apenas uma tecnologia fugiu a esse comportamento, que foi o Lego Mindstorms utilizado em 4 trabalhos.

\subsection{QP4 - Como os alunos deficientes são avaliados no Ensino de Programação?}

Uma questão delicada no ensino é o processo de avaliação dos alunos, que precisa ser bem planejado para que possa efetivamente mostrar ao professor se de fato o conteúdo foi assimilado. Neste mapeamento, identificamos que ainda não existem métodos de avaliação claros para pessoas com deficiência em ensino de programação. A maioria dos trabalhos utiliza como parâmetro as impressões pessoais dos alunos para identificar se a abordagem utilizada foi bem aceita, o que foi feito através de questionários, entrevistas e observação.

Apenas um trabalho catalogado mostrou uma forma de avaliação diferente, Stefik et al. [Stefik et al. 2011] adaptaram a escala inicialmente proposta por Askar e Davenport [Askar and Davenport 2009], que busca medir a autoeficácia em programação Java. No caso do trabalho de Stefik et al. [Stefik et al. 2011], a escala foi adaptada de forma trivial para a linguagem Hop, que foi a utilizada no trabalho. 
IX Congresso Brasileiro de Informática na Educação (CBIE 2020)

Anais do XXXI Simpósio Brasileiro de Informática na Educação (SBIE 2020)

Tabela 1. Tecnologias utilizadas

\begin{tabular}{|c|c|l|}
\hline Tecnologia(s) & $\#$ & Referências \\
\hline \hline ABTB & 1 & [Konecki et al. 2016] \\
\hline Adobe Connect, Google Docs & 1 & [Chiou and Young 2017] \\
\hline BJC Unit, Code.org & 1 & [Wille et al. 2017] \\
\hline Bonk & 1 & [Kane et al. 2018] \\
\hline C++ & 1 & [Connelly 2010] \\
\hline Code.org & 2 & [Stefik et al. 2019], [Wille et al. 2016] \\
\hline HTML,CSS e JavaScript & 1 & [Kearney-Volpe et al. 2019] \\
\hline JAWS & 1 & [Pereira et al. 2018], [Connelly 2010] \\
\hline Pascalzin & 1 & [Pereira et al. 2018] \\
\hline LIBRAS & 2 & [Oliveira et al. 2015], [Granada et al. 2018] \\
\hline Ruby & 1 & [Kane and Bigham 2014] \\
\hline Não definida & 1 & [Capovilla et al. 2015] \\
\hline UDL & 3 & [Israel et al. 2020], [Koushik and Kane 2019], [Ray et al. 2018] \\
\hline SodBeans+Hop(Quorum) & 1 & [Ladner and Stefik 2017], [Stefik et al. 2011] \\
\hline Torino & 2 & [India et al. 2019], [McMillan and Rodda-Tyler 2016] \\
\hline Scratch & 2 & [Koushik and Kane 2019], [Munoz et al. 2018] \\
\hline StoryBlocks & 1 & [Koushik et al. 2019] \\
\hline SuperLOGO & 1 & [Granada et al. 2018] \\
\hline Lego Mindstorms & 4 & [Conchinha et al. 2015], [Ludi and Reichlmayr 2011], [Ludi et al. 2018], \\
\hline & & [Granada et al. 2018] \\
\hline Phogo, Phyton & 1 & [Gonzalez-Sacristan et al. 2016] \\
\hline Legos & 1 & [Capovilla et al. 2013] \\
\hline PAC Mate & 1 & [Connelly 2010] \\
\hline Java & 2 & [Eiselt and Carter 2018], [Ludi and Reichlmayr 2011] \\
\hline
\end{tabular}

\subsection{QP5 - Quais os problemas em aberto na Acessibilidade em Ensino de Programação?}

O mapeamento realizado comprovou que existem estudos relevantes na área, porém ainda existe um vasto campo de pesquisa a ser explorado. Alguns problemas em aberto foram identificados: (i) Como avaliar a aprendizagem em programação de um aluno com deficiência? (ii) Como adaptar exercícios de programação para alunos com deficiência? (iii) Qual metodologia de ensino é adequada para esse público?

Todos esses problemas em aberto precisam levar em consideração que cada tipo de deficiência possui suas especificidades, o que leva a crer que, quando da realização de pesquisas na área, esse é um fator de extrema prioridade.

\section{Discussões}

Identificamos com este MSL que existem diversas pesquisas buscando auxiliar pessoas com deficiência a aprender programação. Como cada tipo de deficiência exige um estudo específico, poucos foram os trabalhos que não restringiram claramente suas pesquisas para um tipo específico de deficiência.

A maioria dos trabalhos utilizou como estudo de caso deficientes visuais e, mesmo para esse público alvo, ainda há muito a ser explorado. Foi observado que para esse tipo de aluno é muito importante a presença de um tutor, de forma a auxiliá-lo nas atividades [Connelly 2010]. No caso de alunos surdos, foi visto que estes possuem mais dificuldade em raciocínio numérico e no conceito de variáveis, sendo um questionamento pertinente analisar quais as melhores formas de abordagem desse tipo de conceito para esses alunos [Granada et al. 2018].

Alunos autistas foram estudados em alguns trabalhos, sendo analisado que associar o ensino de programação ao treinamento de habilidades sociais pode ser benéfico 
IX Congresso Brasileiro de Informática na Educação (CBIE 2020)

Anais do XXXI Simpósio Brasileiro de Informática na Educação (SBIE 2020)

para eles [Eiselt and Carter 2018].

\subsection{Abordagens e Adaptações}

A maioria dos trabalhos encontrados no mapeamento utilizou o que chamamos de Programação Adaptada, que seria utilizar uma mediação específica para que o aluno com deficiência conseguisse acessar as informações e produzir seus códigos de programação. Por exemplo, para alunos cegos, a ideia da programação auditiva pode auxiliar na construção dos programas [Stefik et al. 2011]. Já para alunos surdos, o uso de adaptação por linguagens de sinais se torna interessante, já que para boa parte das pessoas surdas a língua de sinais é sua primeira língua, mas para isso é necessária a criação de glossários de termos técnicos sinalizados, ou seja, assim como na tradução em qualquer linguagem, deve-se ter o cuidado de traduzir determinado termo associado ao seu contexto. Entretanto, mesmo a língua de sinais pode sofrer com regionalismos e isto deve ser levado em conta quando da criação dos sinais técnicos. Existem iniciativas para criação de sinais internacionais, como o criado colaborativamente no ASL-STEM Forum ${ }^{2}$ [Oliveira et al. 2015, Granada et al. 2018].

Alunos com diferenças de aprendizagem foram também analisados e concluiu-se que a barreira mais comum para a aprendizagem de programação foi relacionada à linguagem utilizada [Wille et al. 2017], sendo importante investigar quais adaptações na linguagem técnica poderiam melhorar a assimilação por parte desses alunos. Alunos autistas por exemplo, possuem melhor assimilação com o concreto e o explícito, então Stuurman et al. [Stuurman et al. 2019] afirmam que deve-se abordar os conteúdos exigindo-se o mínimo de contexto possível para entender o que se quer dizer.

\subsection{Replicabilidade de Experimentos}

Lung [Lung et al. 2008] afirma que a replicação é um importante fator para verificação de dados empíricos, desempenhando um papel essencial no avanço do conhecimento científico. Entretanto, ele também concluiu que é difícil e em alguns casos até inconveniente executar replicações literais envolvendo seres humanos na área de computação. Observando os trabalhos encontrados nesse MSL identificamos que as características dos estudantes, os diferentes graus de deficiência e o conhecimento dos pesquisadores poderiam influenciar substancialmente os resultados encontrados.

\subsection{Ameaças à Validade}

Esta pesquisa possui ameaças à validade. O conjunto de artigos retornados podem ser afetados pelas bases e pela chave de busca, bem como por fatores humanos na catalogação dos dados. Outro fator de ameaça à validade foi não selecionar artigos de outras línguas. Ao calibrar a chave de busca, percebeu-se que alguns artigos relevantes não utilizavam termos mais genéricos relacionados ao tema, como "disabilities”, em seus títulos, palavraschave ou resumos. Portanto, foram incluídos termos específicos de algumas deficiências que podem causar algum impacto nos resultados.

\section{Considerações Finais}

Aprender programação já é considerado complexo por diversos fatores e quando se trata de alunos com deficiência a preocupação com o processo de ensino-aprendizagem se torna

\footnotetext{
${ }^{2}$ https://aslstem.cs.washington.edu/
} 
maior. Neste contexto, este trabalho realizou um mapeamento sistemático de literatura com o objetivo de obter uma visão geral de como os pesquisadores e professores estão trabalhando o ensino de programação para esses alunos.

A maior parte dos trabalhos relatou experiências com ensino de programação para pessoas com deficiência visual, do ensino fundamental e médio e adaptando o uso de materiais e tecnologias com auxilio auditivo de um leitor de tela. Os trabalhos em sua maioria utilizavam abordagens e tecnologias diferentes, o que mostra uma ausência de padronização mesmo se tratando de um mesmo tipo de deficiência. O que vimos de forma recorrente nos artigos catalogados é a real necessidade de se adaptar os métodos de ensino de programação tradicionais para atender os diferentes tipos de alunos.

Como oportunidades para trabalhos futuros identificamos algumas vertentes interessantes como a definição de metodologias de ensino adequadas aos diferentes tipos de deficiência, levando em consideração como promover autonomia desses estudantes, respeitadas às devidas limitações. Outra possibilidade é estudar formas efetivas e sistemáticas de avaliação, de forma a saber de fato quem está aprendendo.

Por último, fazemos os seguintes questionamentos: seria possível ensinar programação para pessoas com deficiência de forma que estes alunos saiam de seus cursos devidamente capacitados para, por exemplo, adentrar o mercado de trabalho? É possível padronizar metodologias de ensino-aprendizagem, tecnologias e avaliações levando em consideração as especificidades de cada tipo de deficiência?

\section{Referências}

Al-Ratta, N. M. and Al-Khalifa, H. S. (2013). Teaching programming for blinds: A review. In Fourth ICTA'2013, pages 1-5.

Askar, P. and Davenport, D. (2009). An investigation of factors related to self-eficacy for java programming among engineering students. The Turkish J. of Educ. Tech., 8.

Capovilla, D., Krugel, J., and Hubwieser, P. (2013). Teaching algorithmic thinking using haptic models for visually impaired students. In Proc. of the LaTiCE'13, pages 167171.

Capovilla, D., Mühling, A., and Hubwieser, P. (2015). How learning styles in cs can foster inclusion of visually impaired students. In Proc. of the LaTiCE'15, pages 187-192.

Chiou, P. T. and Young, G. S. (2017). Implementing recommendations of accessibility technology guidelines - the quantitative effects and benefits it offers to non-disabled students. In Proceedings of the CSCI'17, pages 1137-1142.

Conchinha, C., Osório, P., and de Freitas, J. C. (2015). Playful learning: Educational robotics applied to students with learning disabilities. In Proceedings of the SIIE'15, pages $167-171$.

Connelly, R. (2010). Lessons and tools from teaching a blind student. J. Comput. Sci. Coll., 25(6):34-39.

Eiselt, K. and Carter, P. (2018). Integrating social skills practice with computer programming for students on the autism spectrum. In Proc. of the FIE'18, pages 1-5.

Gomes, A. and Mendes, A. (2015). À procura de um contexto para apoiar a aprendizagem inicial de programação. Educação, Formação Tecnologias - ISSN 1646-933X, 8(1). 
IX Congresso Brasileiro de Informática na Educação (CBIE 2020)

Anais do XXXI Simpósio Brasileiro de Informática na Educação (SBIE 2020)

Gonzalez-Sacristan, C., Garcia-Saura, C., and Molins-Ruano, P. (2016). Phogo: A low cost, engaging and modern proposal to learn how to program. In Proceedings of the $I V$ TEEM '16, page 67-71.

Granada, R. P., Barwaldt, R., and Espíndola, D. B. (2018). Glossary of computational terms as a stimulus to programming logic: a case study with deaf students. In Proceedings of the FIE' 18 , pages 1-7.

IBGE (2010). IBGE | Censo 2010. [Online; accessed 3. Jul. 2020].

India, G., Ramakrishna, G., Bisht, J., and Swaminathan, M. (2019). Computational thinking as play: Experiences of children who are blind or low vision in india. In Proceedings of the 21st ASSETS'19, page 519-522.

Israel, M., Jeong, G., Ray, M., and Lash, T. (2020). Teaching elementary computer science through universal design for learning. In Proc. of SIGCSE '20, page 1220-1226.

Kane, S. K. and Bigham, J. P. (2014). Tracking @stemxcomet: Teaching programming to blind students via $3 \mathrm{~d}$ printing, crisis management, and twitter. In Proceedings of the 45th SIGCSE'14, page 247-252.

Kane, S. K., Koushik, V., and Muehlbradt, A. (2018). Bonk: Accessible programming for accessible audio games. In Proceedings of the 17th IDC'18, page 132-142.

Kearney-Volpe, C., Hurst, A., and Fitzgerald, S. (2019). Blind web development training at oysters and pearls technology camp in uganda. In Proceedings of the 16th W4A '19.

Konecki, M., Ivković, N., and Kaniški, M. (2016). Making programming education more accessible for visually impaired. In Proc. of the 39th MIPRO'16, pages 887-890.

Koushik, V., Guinness, D., and Kane, S. K. (2019). Storyblocks: A tangible programming game to create accessible audio stories. In Proceedings of the 2019 CHI '19, page 1-12.

Koushik, V. and Kane, S. K. (2019). It broadens my mind: Empowering people with cognitive disabilities through computing education. In Proc. of CHI '19, page 1-12.

Ladner, R. E. and Stefik, A. (2017). Accesscsforall: Making computer science accessible to k-12 students in the united states. SIGACCESS Access. Comput., (118):3-8.

Ludi, S., Bernstein, D., and Mutch-Jones, K. (2018). Enhanced robotics! improving building and programming learning experiences for students with visual impairments. In Proceedings of the 49th SIGCSE'18, page 372-377.

Ludi, S. and Reichlmayr, T. (2011). The use of robotics to promote computing to precollege students with visual impairments. ACM Trans. Comput. Educ., 11(3).

Lung, J., Aranda, J., Easterbrook, S., and Wilson, G. (2008). On the difficulty of replicating human subjects studies in software engineering. In In Proceedings of the 30th ICSE'08, pages 191-200.

Martin, C. S. (2009). Os fundamentos das deficiências e síndromes - Aprender e superar. [Online; accessed 3. Jul. 2020].

McMillan, C. and Rodda-Tyler, A. (2016). Collaborative software engineering education between college seniors and blind high school students. In Proceedings of the 38th ICSE' 16, page 360-363. 
Munoz, R., Villarroel, R., Barcelos, T. S., Riquelme, F., Quezada, A., and BustosValenzuela, P. (2018). Developing computational thinking skills in adolescents with autism spectrum disorder through digital game programming. IEEE Access, 6:6388063889.

Oliveira, F. C. d. M., Gomes, G. N., de Freitas, A. T., de Oliveira, A. C., Silva, L. C., and Queiroz, B. (2015). A comparative study of the acceptability of signs for the brazilian sign language created in person and remotely. In Proc. of SIGCSE '15, page 207-211.

Pereira, R. M., da Silva, F. F., and Silla, C. N. (2018). Teaching algorithms for visually impaired and blind students using physical flowcharts and screen readers. In In Proceedings of the FIE'18, pages 1-9.

Petersen, K., Feldt, R., Mujtaba, S., and Mattsson, M. (2008). Systematic mapping studies in software engineering. In Proceedings of the 12th EASE'08, page 68-77.

Ray, M. J., Israel, M., Lee, C. e., and Do, V. (2018). A cross-case analysis of instructional strategies to support participation of k-8 students with disabilities in cs for all. In Proceedings of the 49th SIGCSE' 18, page 900-905.

Silva, T., Medeiros, T., Medeiros, H., Lopes, R., and Aranha, E. (2015). Ensinoaprendizagem de programação: uma revisão sistemática da literatura. Revista Brasileira de Informática na Educação, 23(01):182.

Souza, D., Batista, M., and Barbosa, E. (2016). Problemas e dificuldades no ensino de programação: Um mapeamento sistemático. Revista Brasileira de Informática na Educação, 24(1):39.

Stefik, A., Ladner, R. E., Allee, W., and Mealin, S. (2019). Computer science principles for teachers of blind and visually impaired students. In Proceedings of the 50th SIGCSE' 19, page 766-772.

Stefik, A. M., Hundhausen, C., and Smith, D. (2011). On the design of an educational infrastructure for the blind and visually impaired in computer science. In Proceedings of the 42nd SIGCSE' '11, page 571-576.

Stuurman, S., Passier, H. J., Geven, F., and Barendsen, E. (2019). Autism: Implications for inclusive education with respect to software engineering. In Proceedings of the 8th CSERC'19, page 15-25.

Wille, S., Century, J., and Pike, M. (2016). Computer science principles (csp) and students with learning differences: Expanding opportunities for a hidden underrepresented group. In In Proceedings of the RESPECT'16, pages 1-8.

Wille, S., Century, J., and Pike, M. (2017). Exploratory research to expand opportunities in computer science for students with learning differences. Computing in Science Engineering, 19(3):40-50. 